\title{
UniMAP e-Lab for Electrical Engineering Technology: Future Online Laboratory Classes
}

\author{
Mohd Hisam Daud ${ }^{1, *}$, and Zol Bahri Razali ${ }^{1}$ \\ ${ }^{1}$ Robotics and Automation Technology, Faculty of Engineering Technology, Universiti Malaysia \\ Perlis, 02100 Padang Besar, PERLIS, MALAYSIA
}

\begin{abstract}
This paper will describe a proposed design and approaches to the future provision of laboratory experience using communication and control technology. This approach provides broad access for students who can not attend conventional laboratory to laboratory work. The experimental system online (e-Lab) is under development for the students to carry out various experiments in engineering and technology education assessment system. In the field of engineering technology, one important element is laboratory work, although there are limitations in terms of space laboratories, distance learning provision. UniMAP e-Lab project aims to address many of the existing constraints. Beyond the educational goals, UniMAP e-Lab system enables experimental knowledge in a particular field of engineering technology and experimental results of the research are disseminated and exploited effectively. Solution design of hardware and software as well as the characteristics of education discussed.
\end{abstract}

\section{Introduction}

This paper discussed the development of UniMAP e-Lab system (or known as remote laboratory) to enable students to carry out experiments as an extension of the real world required a lot of distance learning systems and computer-based learning. The objective of this system is to provide a high quality learning experience in engineering technology education with a teaching laboratory for students [1], provides flexibility in terms of location, special needs and time. The project which is developing an online experimentation system, will allow students to conduct a range of experiments to demonstrate the utility of such an approach for engineering technology education [2].

For instance, currently the PEARL system will allow students to work together while at a distance from the laboratory site using a range of synchronous and asynchronous communications tools. Experiments developed include an implementation of a remote electron microscope, a spectrometer, visual inspection of printed circuit boards and a digital electronic bench. A key design feature of this web-based system is that the interface should be compatible with assistive technology [3].

Remote access laboratories have been widely reported by researchers and education innovators since the internet started to provide reliable data communications and easy access for large numbers of users. However, most reports in the contemporary literature

\footnotetext{
* Corresponding author: mhisam.daud@gmail.com
} 
describe technical implementations without extended and detailed evaluation of student learning advantages. So far, it would appear, no detailed cost benefit studies have been reported. It would seem that the technology has stalled [4]. Early implementations, such as Telelabs at the University of Western Australia (UWA), are in regular operation for large classes of students [4]. Yet the technology does not seem to have been widely adopted, nor have there been reports of serious attempts to negotiate industry standards that would be a necessary precursor for large-scale adoption.

Why has such a promising technology not been adopted so readily? Are the benefits as great as proponents have claimed in the past?

\section{Literature Review}

\subsection{Effectiveness of the e-Lab.}

When evaluating the effectiveness of the learning process in engineering technology education, practical experience is one of the outcomes. Hands-on laboratories play an important role not only with respect to this practical aspect but also when talking about developing student's creativity and problem solving skills [5]. So that, in the past 10 years, many research involved in remote laboratory research to measure the experience of students.

A report by Trevelyan and his colleague [4] on a fundamental investigation of some learning phenomena in conventional hands-on laboratories that arose from the question "What do students really learn in laboratory classes or remote lab experiences?". To explore this issue, the authors started a project in order to understand more about the results of practical learning in the traditional classroom lab. The notion of a group of researchers, to settle practical matters related to the specific domain it depends on the ability of someone in 'practical intelligence' (PI). Psychologists develop PI measurement instruments as part of the whole debate continued discipline predict performance on-the-job who use the results of psychometric tests. The study found that in laboratory-duty pliers, they can measure the significant gains in PI and these techniques can be used. Conventional evaluation (lab reports, tests, exams, training tutorials) obtained from the results of students not associated with PI. Even more interesting, perhaps through PI, fault diagnosis tasks to be perform can be predicted by the ability of students and their found this evidence.

For the first time, therefore, the researchers demonstrated that there are real advantages inherent in hands-on laboratory classes and we can measure this advantage [2]. What can be learned from this work is that students' learning in laboratory classes is not what the lecturer have come to expect. There is still much to discover and the report provided some research tools to enable others to follow similar investigations.

There are significant implications for developers of UniMAP e-Lab system and engineering technology education researchers from this work.

\section{2 e-Lab: Evaluating Students Learning Experience.}

In 2000, UWA decided to build on the Telelabs project to combine automated assessment and performance monitoring techniques with internet-connected remotely operable laboratory equipment to enhance student learning and provide a real return on investment through savings in laboratory demonstrator time [4].

This research shows how through the dial-up phone and internet connection, one or more users can use low-bandwidth connections simultaneously operated successfully by a complex machine. This research project and other projects that have stimulated using an 
internet connection for a whole generation of remote control equipment. The industrial automation and control equipment is now standard for most remote internet control for the hardware and software equipment.

Online access to lab equipment have given more time for students to interact with our equipment: conventional laboratory classes usually allows each student only a few minutes real time operating equipment [5]. By allowing students to operate the equipment for an hour or more at a time, and to collect data by means of semi-automatic, we have significantly improved opportunities for student learning.

At the same time, to improve the students learning technique, the researchers have also attempted an incentives to measure this through develop assessment techniques. Of course, real equipment should always be expose to students [5]. The question still needs to answer is "how much time needed for supervision in the real laboratory with real equipment can be replaced with remote access without supervised?"

In evaluating the engineering technology laboratory class effectiveness, student evaluations or opinions may also be included [6]. Explicit learning outcomes, usually written in a handout guide for a mechatronics laboratory class, create intentionally: students perform mechatronics laboratory exercises in order to learn these. Implicit learning, on the other hand, may not be intentional.

For example, in Robotics and Automation laboratory class setting, students will learn more than just the stated objectives of the class. Through their experience they will learn many other things that may not seem to be important for gaining marks, but nevertheless are helpful in achieving a commonly desired objective for students: completing the class as quickly as possible [2]. In an electronics laboratory, for example, this learning might include convenient ways to strip connecting wires, ways to make reliable electrical connections, etc. This is what we refer to as practical knowledge, and one way to measure this learning is through the notion of practical intelligence [4].

Measuring the hands-on components of laboratory classes might be valuable for engineering technology educators. Before moved from hands-on to e-laboratories, engineering technology educators need to justify the experiences of certain students when the measurement is validated.

Through a platform of assessment in the e-laboratory, learning component can be distinguished by measuring and assessing it. The educator could be more confident in predicting what students will gain in a given laboratory class [1]. These measuring instruments would then provide a powerful new means to separate between e-laboratories and hands-on and also to assess the effectiveness of engineering technology laboratory classes in UniMAP.

\section{Methods and materials}

\subsection{Existing technology for e-Lab.}

Nowadays the e-Laboratories technologies already been used. The e-Laboratory (or remote laboratory) is defined as a computer-controlled laboratory that can be accessed and controlled through some communication medium. Different architectures are used to support the e-learning environments. Some are based on software solutions such as LabVIEW, while others are supported by open-source software such as PHP, JavaScript, Java, Pearl, Python, AJAX.

e-lab allows the user to perform tasks and laboratory experiments without having to be or using real equipment via the Internet. In a traditional laboratory, consumers use equipment directly through physical action (eg manipulate by hand, switch button, pressing 
the button) and receive sensory feedback (visual, audio and tactile). In the e-lab, the same interactions occur just like being in the lab either in the wall or around the world, via the remote infrastructure. Remote infrastructure is a new layer of communication between the users of laboratory equipment. It is responsible for receiving sensory information from the equipment and convey the user's actions.

Therefore, students will have access to laboratory facilities without restrictions on time and location. Remote access is the application of laboratory in which students connect to a remote laboratory equipment, carry out experiments and get the results of that experiment as a response [7]. It introduces students to the physical remote laboratory provides physical and environmental practices that students can use the web interface from any computer [8].

So in this section is discussed a few types of e-laboratories with different architecture and tools used to develop them

\subsection{LabVIEW web UI builder.}

Unlike traditional web programming languages, LabVIEW Web UI Builder enables engineers and scientists to use their existing knowledge of LabVIEW graphical programming in order to develop web applications. National Instrument Web UI Builder is an online editor based on Microsoft's Silver Light Framework that provides the user a LabVIEW like experience for developing remote application based on the LabVIEW RESTful web services. Web services are available as a development option for LabVIEW application from version 8.6 and were introduced to address the growing need for an open standard way to interact with LabVIEW application over the Web. Thin client application created with LabVIEW Web UI Builder, in combination with LabVIEW web services, provide remote monitoring and control solutions that are based on modern, standard web technologies. These solutions do not depend on the LabVIEW Run-Time Engine, and all network communication performed through the standard Hypertext Transfer Protocol (HTTP) [8].

Furthermore, remote control applications can be created using LabVIEW Web UI Builder. Remote control easily implemented when only one end user via a thin client that will connect and use the system. during the development of thin clients, it is necessary to take into account if the end user is more than one. Solutions that LabVIEW Web UI Builder users can perform include requiring verification as part of the login or use a web service to track the number of users currently communicating with the system.Figure-1 shows an appearing monitor of LabVIEW Web UI Builder [8].

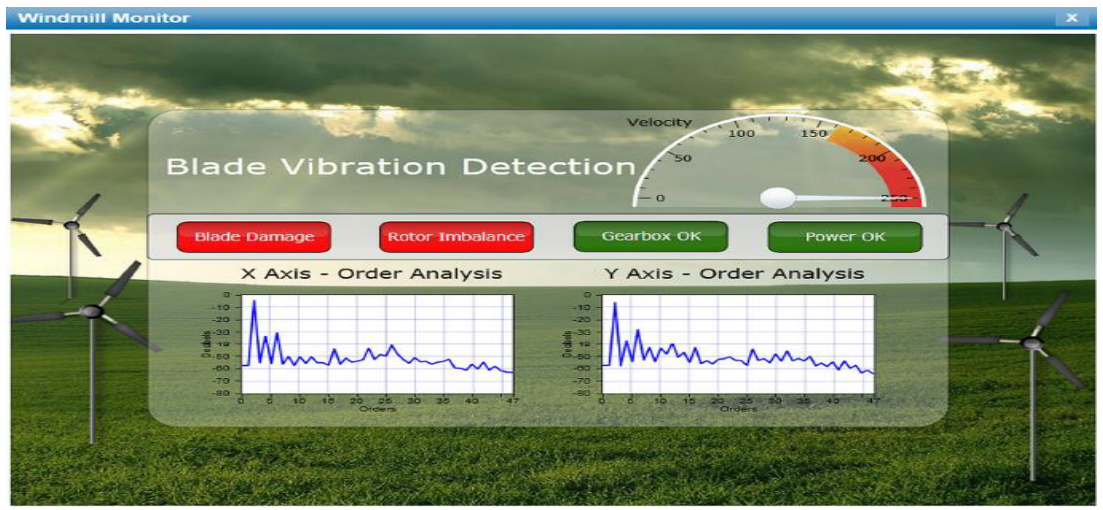

Fig. 1. LabVIEW Web UI Builder 


\subsection{Smartphone and cross-platform communication toolkit (SCCT).}

Smartphone and Cross-Platform Communication Toolkit (SCCT) is a programming toolkit supported on a wide range of platforms and programming languages that manages communication among application transparently and speeds up your delivery time. SCCT create open solutions capable of exchanging data among a wide range of systems from smartphones to Java apps and UNIX workstations. The SCCT transparently manages communication between applications so you can create multiple simultaneous connections without changing a single line of code. Data is converted into a platform-independent format and optimizes data packing to get the best data transfer performance. It also supported on windows, android, Linux, iOS, and phone7 for different programming languages [9].

The publisher-subscriber architecture Remote Lab is realized using the library provided by SCCT Communication in LabVIEW. library publisher is to create a complete publisher, which confirms the customer in, check the status of a connection and send data to all active publishers and pass their requests to the application. Publishers maintain server side communication system. Client library is to create customers who handle all the details of communication with the publisher. It received the package data and present them in front end of the application according to their data type.

SCCT facilitate the exchange of data between applications and handle a lot of connections at a time and SCCT facilitate the exchange of data between applications and handle many connections at the time and publish data in different formats according to the client's ability. Furthermore, SCCT provides high-performance data communications on conventional platforms such as HMTL5, JavaScript, Android, LabVIEW, Java, [9], thus making it a multi-platform approach.

\subsection{WebLab-deusto.}

University of Deusto had developed the remote lab management system that call WeblabDeusto. In both versions the beginning it was an ad hoc remote laboratories [10] which is far above the lab system that can be built different laboratories using different software technology and currently supports federal laboratories far. The WebLab-Deusto architecture (see Figure 2) is a client-server architecture. Thus, this the intensity of light is the factor of the other fireflies to move toward the other firefly.

The average student interacts with server login for confirmation and with server core to during operation. Server core will operate truth, tabulation and information storage sent by students, and submit a all requests to laboratory that is correct. Behind core server, WebLab-Deusto architecture defined two more waiter is lab server and experiment server.

The first is to be placed in a physical laboratory where various experiments Servers expected. The purpose is mainly to provide security Experiment Servers, and also periodically check if it still works, and acts as a wrapper. Experiments Server is a server that physically interact with the hardware. Library to build Experiment Server is available for different platforms $(\mathrm{C}++, \mathrm{C}$, Java, Python, Labview). All interaction between the client and the server are sent via a web service. Either SOAP, JSON over HTTP and XML-RPC can be used. Two types of laboratories can be built using WebLab-Deusto laboratory managed and unmanaged

In managing the laboratory, all interaction between students and laboratory is managed through all the layers described. Therefore, the savings made on Server Core and Server Experiments must not be common at the network level. However, protocols based on directives must be implemented in each laboratory. In laboratory unmanaged, Experiment existing server to manage other platforms [10] as a Virtual Machine or LabVIEW Remote 
Panel. Thus, the student is still interacting with all levels, but the main interaction is controlled by external platforms (eg Virtual Machines with VNC or Remote Desktop), and it is not stored or securized by WebLab-Deusto.

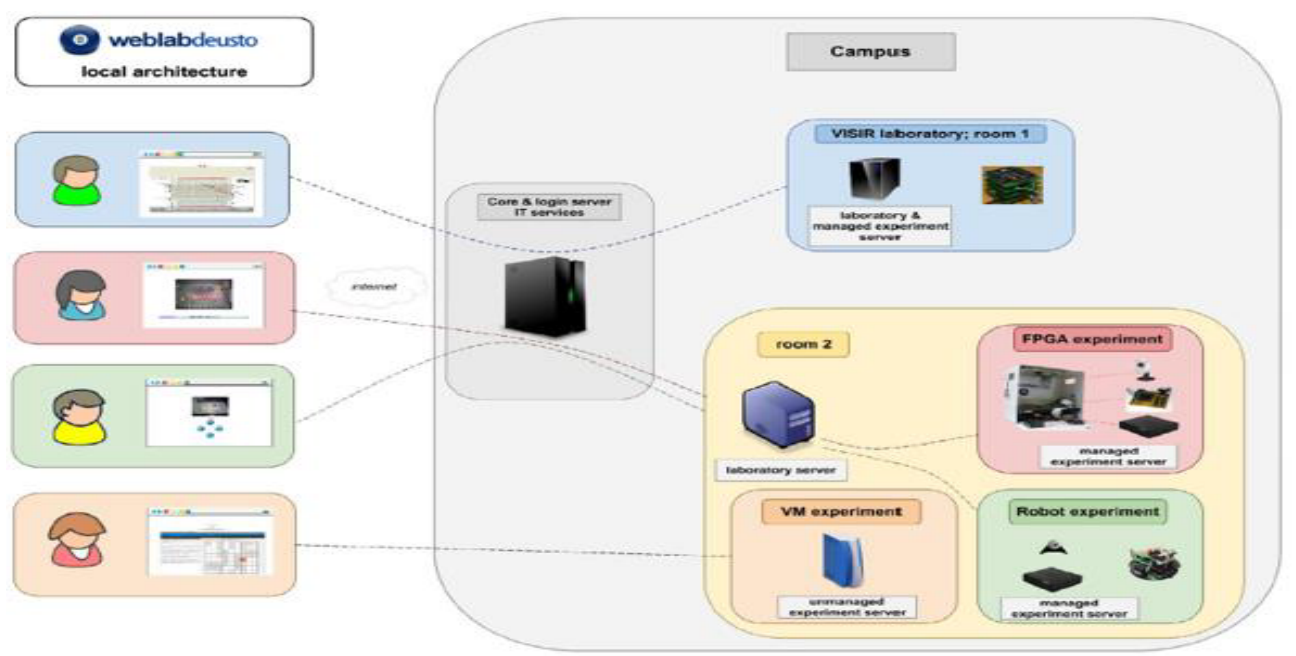

Fig. 2. The WebLab-Deusto Architecture

\section{Results and discussions}

\subsection{Method of developing e-Lab.}

This section has described the method of developing the e-laboratory system. The system divided into software and hardware. The most problems in developing the system are software developers (develop server: web server, database server and application server). It also needs a skill in programming such as $\mathrm{C}, \mathrm{C}++$, Java, NET, Python, etc.

Figure 3 shows that the general of e-laboratory architecture that divides into a few parts. In this architecture, clients connect to the core and login servers, using common HTTP. The users may use a personal computer (PC), laptop, hand phone (android, iOS, etc.), etc. to control the lab as long as there has internet connection. A web server is a program that uses client or server model and the World Wide Web HTTP serves files that make up a web page for web users (the computer containing the HTTP client that submitted their request).

Every computer needed a web server program to connected to the internet with a website. Web servers often come as part of a larger package of Internet and intranet-related programs to serve e-mail, download requests to the File Transfer Protocol (FTP) file, and building and publishing a website. Consideration in choosing a web server including how it works with the operating system and other server, the ability to control the program-server, security features, and publications, search engines, and tools of building a website that might come with it. This server manages authentication, authorization, user tracking, Federal (share) and scheduling. From there, the system forwards the request to the lab servers (application servers), which pointed to the end of the experiment.

For application waiter have been exposed business logic for customer application through various protocols, probably include HTTP. Although web server especially related to send HTML for display in web browser, application waiter providing access to business logic to be applied by client applications program. Application programme can use this logic just because it will call method in object (or function in procedure world). the 
application waiter customer will include GUI (graphical user interface) that walk in PC. Information that commute between application waiter and the client is unlimited to markup easy display. On the other hand, information that is logical programme.

Since the logic takes the form of data and method calls and not static HTML, the client can use the exposed business logic however it wants. In most cases, the server exposes this business logic through a component API, such as an EJB (Enterprise JavaBean) component model found on J2EE (Java 2 Platform, Enterprise Edition) application server. In addition, the application server manages its own resources. The task of keeping the door including security, transaction processing, resource gathering, and messages.

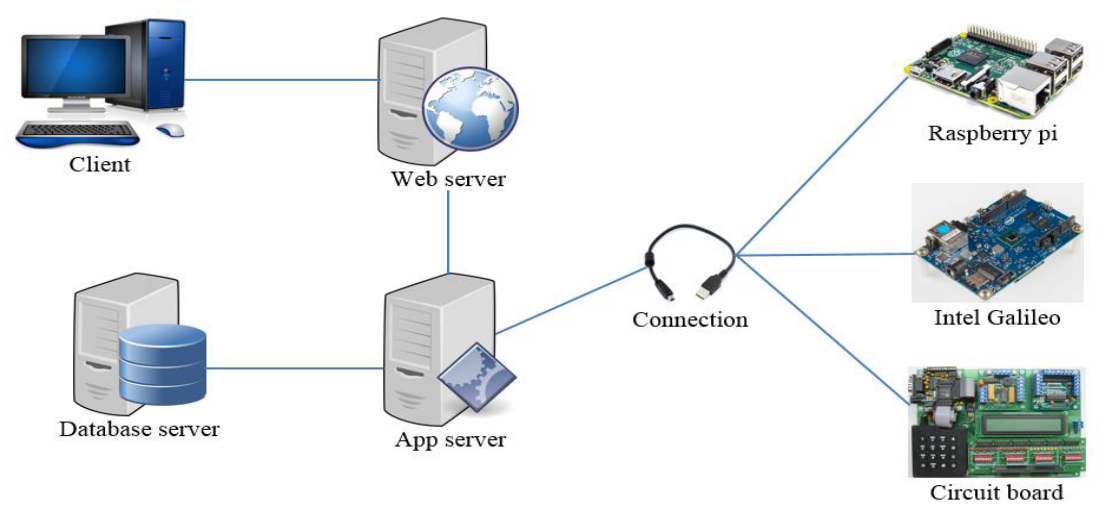

Fig. 3. The UniMAP e-Lab Architecture

The connection between the application server with instrument (electronic circuit, control system, etc.) is depending on the system. Some system uses serial communication cable (RS232), direct cable (such as Raspberry pie that use the USB cable and connect to the computer), etc. The instrument here is equipment that use in the laboratory and also the experiment that the student will be held. The signal that users send from the client will be sent to this equipment.

\section{Discussions}

Online access to lab equipment have given more time for students to interact with lab equipment: conventional laboratory classes usually allows each student only a few minutes away of equipment operating time. By allowing students to operate the equipment for an hour or more at a time, and to collect data by means of semi-automatic, has improved student learning opportunities significantly.

Apart from the advantage that it provides much more opportune time for students to operate equipment for themselves, the main strength of the UniMAP e-Lab project has been as a centrepiece for the Robotics and Automation Technology degree course. Students in the course have learned mechatronic systems integration and the development of techniques to develop and expand e-Lab system at all faculties of engineering technology. Students in the second and third using this system for practical work because it combines many different elements of robotics and automation.

Although the rapid risen of communication technology, the technology has not been widely adopted for the purpose of education, such as the development of e-Lab, either in our own faculty or elsewhere. Nearly all engineering laboratory classes still follow traditional patterns, as do lecture and tutorial classes. Therefore, it is worth asking why the adoption of such an attractive technology has been so much slower than expected. Many of 
the original researchers that pioneered remote laboratory implementations have moved on to other research or have returned their original research interests.

In our research, early in the project the authors were concerned that a complex LabVIEW-based software application for the server development might require extensive technical support as it migrated through the operating system and computer hardware upgrades. While significant effort has been needed on occasions. Gradually, however, some other significant limiting factors have become apparent.

\section{Conclusion}

Based on the concern about unadopted latest and current communication technologies and opportunities, the authors start to work on the UniMAP e-Lab (online Lab or remote Lab), with the hope to use and get benefit from the technologies. Through our hands-on working and observation, UniMAP e-Lab seem to provide more time for students or repeatly to interact with equipment: of actual time operating equipment. By allowing students to operate the equipment repeatedly, and collect data in the semi automated fashion, have significantly improved opportunities for student learning.

For students Robotics and Automation Technology degree course in UniMAP, apart from the advantage that it provides much more opportunity in laboratory sessions. By extending and develop the e-Lab system, users could learned electronic, electrical and mechanical integration and also system development techniques. Students in the second and third years using this system for practical work because it combines many different elements of robotics and automation.

The e-Lab system, when completed, will be embedded into UniMAP e-Learning LMS (Learning Management System) through e-Learrning platform. However to achieve the goal is still too far: we start from the scratch.

This work was financially supported by the Universiti Malaysia Perlis - Fundamental Research Grant Scheme (9003-00442).

\section{References}

1. Z.B. Razali, L.M. Kamarudin, Blended Learning and Flipped Learning in Malaysia, 9, (UKM, 2014)

2. Z.B. Razali, Advancements in Robotics and Mechatronics, 34 (2015)

3. C. Colwell, E. Scanlon, M. Cooper, Comput. Educ., 38, 65 (2002)

4. J.P. Trevelyan, Z.B. Razali, Internet Accessible Remote Laboratories: Scalable ELearning Tools for Engineering and Science Disciplines, 416 (Engineering Science Reference, 2012)

5. Z.B. Razali, J.P. Trevelyan, Procedia Soc. Behav. Sci., 56, 42 (2012)

6. L.D. Feisel, J.R. Albert, J. Eng. Educ., 94, 121 (2005)

7. D. Cmug, T. Mutapcic, F. Zoino, Remote Versus Classical Laboratory in Electronic Measurements Teaching - Effectiveness Testing, XVIII Imeko World Congress Metrology for A Sustainable Development, Rio de Janerio, (2006)

8. P. Sladek, L. Pawera, J. Valek, Procedia Soc. Behav. Sci., 12, 164 (2011)

9. http://www.toolsforsmartminds.com/products/SCCT.php rec: 21 April 2015

10. P. Orduna, L. Rodriguez Gil, D. Lopez de Ipina, J. Garcia Zubia, Sharing the remote laboratories among different institutions: A practical case, 9th International Conference on Remote Engineering and Virtual Instrumentation (2012) 